\title{
Endoventricular Spiral Plication for Ischemic Dilated Cardiomyopathy
}

\section{Kayo Sugiyama*, Yasuhiro Futamura, Hirotaka Watanuki, Masaho Okada, Genri Tagami, Katsuhiko Matsuyama}

Department of Cardiac Surgery, Aichi Medical University Hospital, Nagakute, Japan

Email: *kayotaro3@gmail.com

How to cite this paper: Sugiyama, K., Futamura, Y., Watanuki, H., Okada, M., Tagami, G. and Matsuyama, K. (2019) Endoventricular Spiral Plication for Ischemic Dilated Cardiomyopathy. Open Journal of Thoracic Surgery, 9, 1-7. https://doi.org/10.4236/ojts.2019.91001

Received: February 13, 2019

Accepted: March 25, 2019

Published: March 28, 2019

Copyright $\odot 2019$ by author(s) and Scientific Research Publishing Inc. This work is licensed under the Creative Commons Attribution International License (CC BY 4.0). http://creativecommons.org/licenses/by/4.0/

\begin{abstract}
Surgical ventricular restoration (SVR) procedures have been developed; however, their long-term effectiveness remains controversial. Although a series of endoventricular spiral plication (ESP) has been rarely reported and its long prognosis is still unknown; this method has a unique concept of left ventricular (LV) restoration without artificial patch materials. Here, we describe the case of a patient with ischemic cardiomyopathy and ischemic mitral regurgitation who successfully underwent ESP, mitral valve repair, and coronary artery bypass grafting. ESP was effective in papillary muscle approximation for avoiding heart failure; however, the noted improvement of LV wall thickening might be temporary.
\end{abstract}

\section{Keywords}

Endoventricular Spiral Plication, Ischemic Cardiomyopathy, Surgical

Ventricular Restoration, Ischemic Mitral Regurgitation

\section{Introduction}

Cardiac transplantation, an accepted treatment option for patients with end-stage cardiac failure, is limited by severe shortages of donor hearts. Surgical ventricular restoration (SVR) affords significant improvement in the ejection fraction, compared with coronary artery bypass alone, without added mortality [1]. Although SVR for ischemic cardiomyopathy (ICM) has been developed and its short-term outcome is reportedly satisfactory, the surgical techniques for SVR are still evolving and their long-term outcome remains uncertain [1] [2] [3]. In patients with ICM, mitral regurgitation (MR) occurs because of dilatation of the mitral annulus and the tethering effect of the mitral leaflet due to dilatation of the left ventricle (LV). Endoventricular spiral plication (ESP) has been reported 
as a less-invasive SVR procedure that does not require artificial prostheses [4] [5]. This procedure can be performed with a small incision of the LV wall and is also expected in papillary muscle approximation with spiral suturing. Furthermore, this procedure is expected to detect another advantage in increase of LV wall thickness. Based on the etiology of MR in patients with ICM, restoration of the LV appears to also restore the tethering of the mitral leaflet by surgical reverse remodeling of the LV. MR is treated by papillary muscle approximation to repair the mitral tethering [1] [6].

\section{Case Report}

A 70-year-old man complained of progressive dyspnea on exertion. He had been treated for hepatitis types B and C and gastric ulcer at a local hospital and had no obvious past history associated with Kawasaki disease. Chest radiography showed cardiomegaly and congestion in the bilateral lungs (Figure 1(a)). Electrocardiography showed complete left bundle branch block (Figure 1(b)). Transthoracic echocardiography showed diffuse LV dilatation with dyskinetic apical motion and moderate MR (Figure 3(a)) due to tethering. The LV end-diastolic diameter was $66 \mathrm{~mm}$; estimated LV end-diastolic and -systolic volumes were 273 $\mathrm{mL}$ and $244 \mathrm{~mL}$, respectively; $\mathrm{LV}$ end-diastolic and systolic volume indexes were $175 \mathrm{~mL} / \mathrm{m}^{2}$ and $156 \mathrm{~mL} / \mathrm{m}^{2}$, respectively; and the $\mathrm{LV}$ ejection fraction was $11 \%$. Coronary angiography revealed total occlusion of the left anterior descending artery (Figure $1(\mathrm{c})$ ). Left ventriculography showed dilatation and diffuse hypokinesis of the LV and a dyskinetic apex. Other blood examinations were almost normal, but the patient's brain natriuretic peptide level was $945 \mathrm{pg} / \mathrm{mL}$.

ESP using cardiopulmonary bypass with cardiac arrest, with concomitant coronary artery bypass grafting to the left anterior descending artery using the left internal thoracic artery and mitral annuloplasty with an artificial ring were performed. Myocardial protection was achieved using antegrade and retrograde cold-blood cardioplegia. After the LV was opened at the apex parallel to the left anterior descending artery, the boundaries between the normal and aneurysmal scar tissues were identified. After LV incision, because the dimension between the bilateral papillary muscles was greater than $3 \mathrm{~cm}$, papillary muscle approximation was performed using pledgeted 3-0 polypropylene stitches with autologous pericardial patch (Figure 2(a)). Afterward, continuous double-spiral suturing using pledgeted 3-0 polypropylene stitches was started $5 \mathrm{~cm}$ proximal to the apex. The stitches were placed spirally at intervals of $2 \mathrm{~cm}$ rotating in a clockwise direction between the basal septum and newly-created LV apex to plicate the ideal LV shape (cone shape) (Figure 2(b)). After the LV plication, the stitches were tied down snugly. The LV was closed without any patch materials with interrupted mattress suturing and reinforced with continuous running suturing using 3-0 polypropylene stitches (Figure $2(\mathrm{c})$ ). The durations of the operation, cardiopulmonary bypass, and aortic cross-clamping were 387,166 , and 73 minutes, respectively. The weaning from the cardiopulmonary bypass was uneventful, and MR decreased to trivial. 


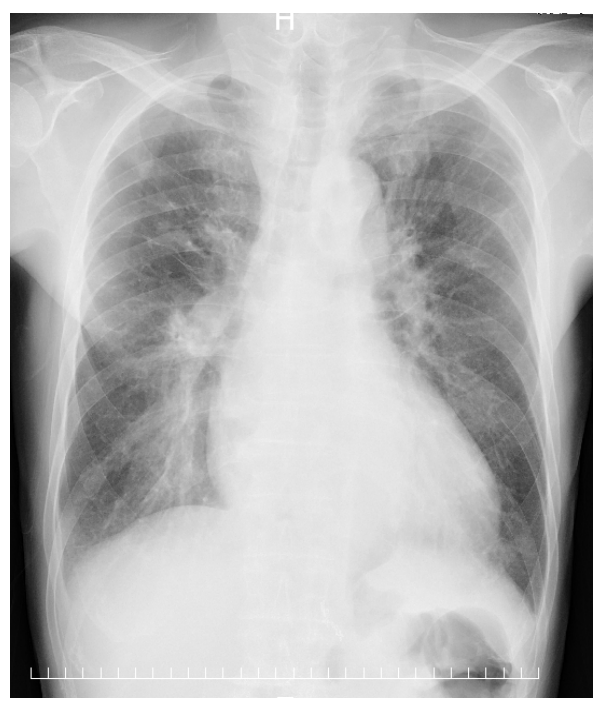

(a)

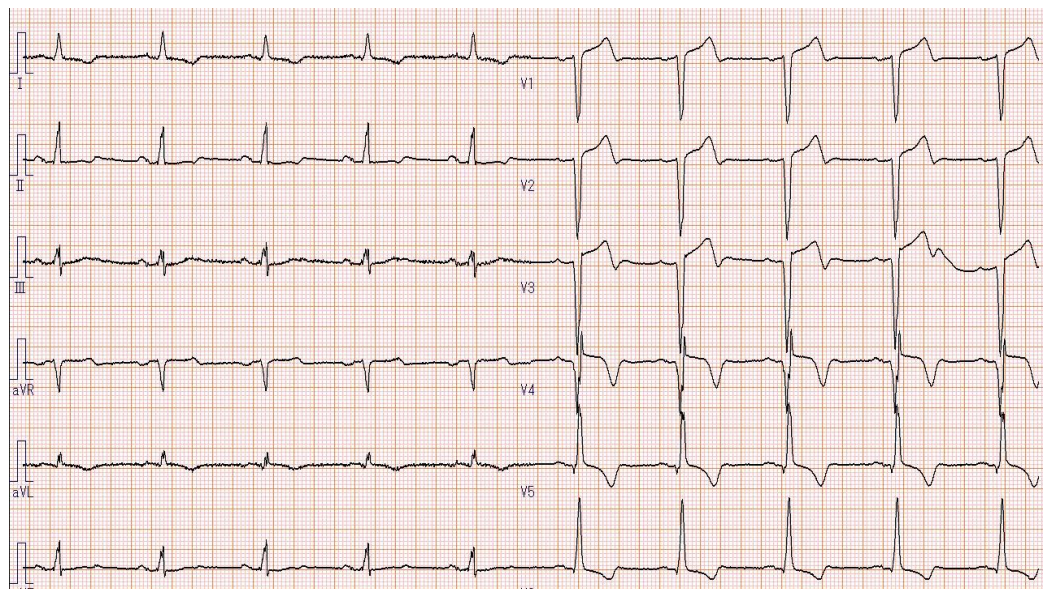

(b)

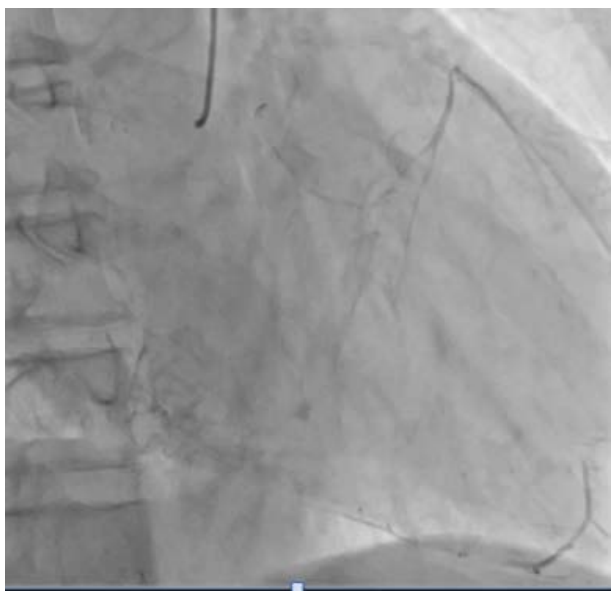

(c)

Figure 1. (a) Preoperative chest radiograph showing cardiomegaly and congestion in the bilateral lungs; (b) Preoperative electrocardiogram showing complete left bundle branch block; (c) Preoperative echocardiography showing a distended left ventricle and moderate mitral regurgitation.

The transthoracic echocardiography performed 1 week postoperatively revealed improvement of LV dilatation and LV shape. The LV end-diastolic diameter was $58 \mathrm{~mm}$ and the estimated LV end-diastolic and -systolic volumes were reduced to $160 \mathrm{~mL}$ and $111 \mathrm{~mL}$, respectively. The LV end-diastolic and -systolic volume indexes improved to $103 \mathrm{~mL} / \mathrm{m}^{2}$ and $71 \mathrm{~mL} / \mathrm{m}^{2}$, respectively, and the LV ejection fraction increased to $31 \%$. It also revealed improvement in thickness of the LV wall; the interventricular septal diameter, posterior wall diameter, and LV mass index increased from 9.4 to $11.6 \mathrm{~mm}, 10.2$ to $11.8 \mathrm{~mm}$, and 221 to $258 \mathrm{~g} / \mathrm{m}^{2}$, respectively (Table 1 ). There was no MR (Figure 3(b)). Postoperative magnetic resonance imaging showed improvement of LV motion and LV shape (Figure 3(c)).

The patient was postoperatively treated with cardioprotective medication including $2.5 \mathrm{mg}$ enalapril, $5 \mathrm{mg}$ carvedilol, and $5 \mathrm{mg}$ pimobendan. At the 1-year 


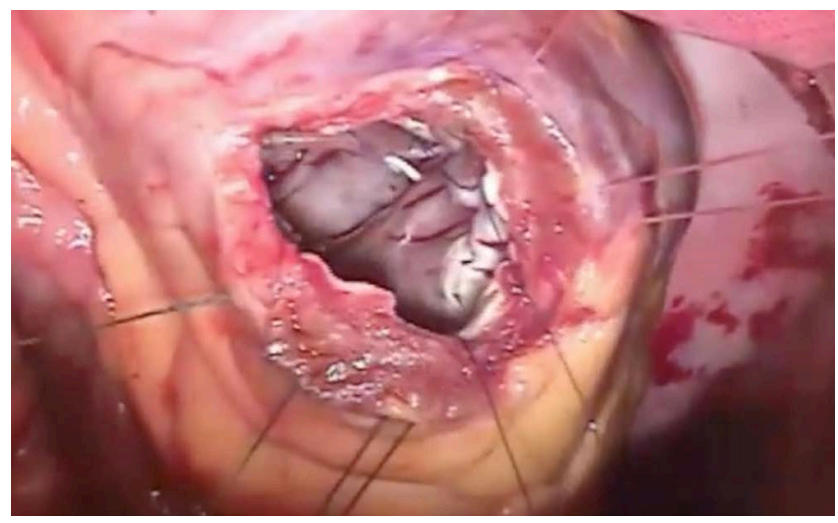

(a)

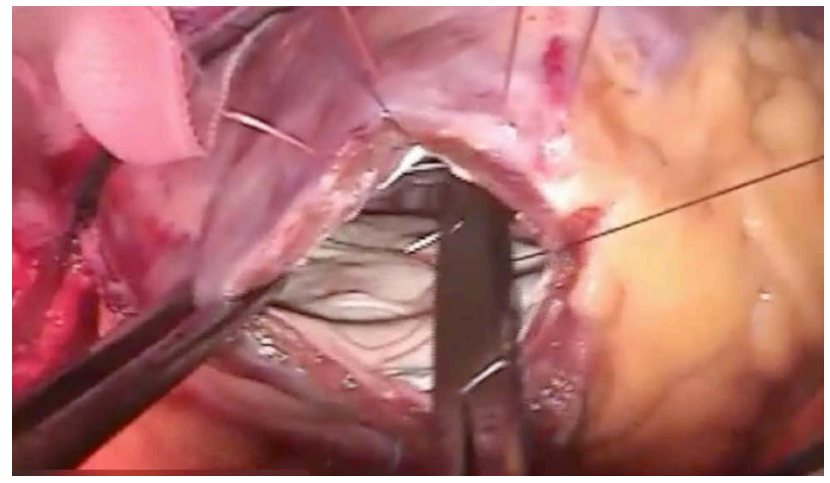

(b)

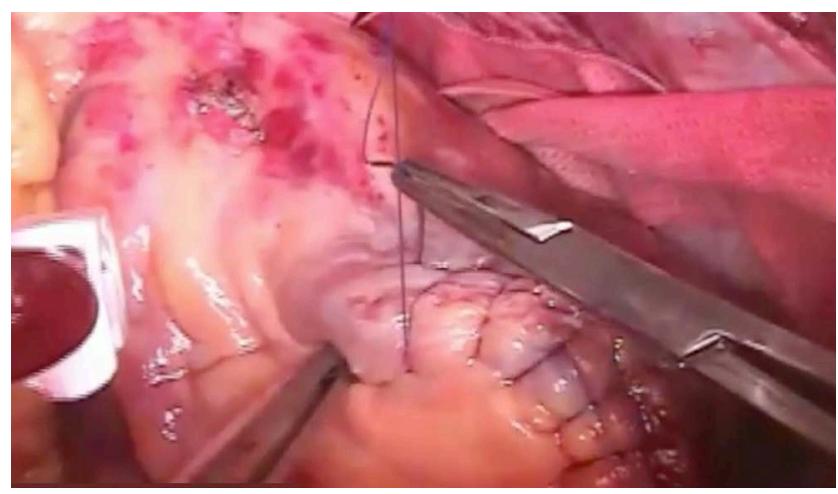

(c)

Figure 2. (a) Intraoperative view showing papillary muscle approximation; (b) Intraoperative view showing stitches placed spirally at intervals of $2 \mathrm{~cm}$ rotating in a clockwise direction between the basal septum and the newly-created LV apex to plicate the ideal LV shape; (c) Intraoperative view showing the left ventricle closed without any patch materials with interrupted mattress suturing and reinforced with continuous running suturing using 3-0 polypropylene stitches.

follow up, the patient's condition was satisfactory without any major adverse cardiac events. However, the echocardiography performed 1 year postoperatively revealed recurrence of LV dilatation, decrease in the LV ejection fraction, and worsening in LV wall thickening compared to the postoperative echocardiogram, whereas there was still no MR (Figure 3(d), Table 1). A longer follow-up of the patient could be conducted in this article.

\section{Discussion}

The SVR strategies for ICM have been evolving. SVR affords significant improvement in the ejection fraction, compared with coronary artery bypass alone, without added mortality [1]. Even thought the clinical effectiveness of SVR remains controversial, SVR could constitute an alternative strategy to heart transplantation for patients with severe ischemic cardiomyopathy. Cardiac transplantation, an accepted treatment option for patients with end-stage cardiac failure, is limited by severe shortages of donor hearts. However, most SVR procedures are highly invasive and require patch materials. Moreover, there is no established method of LV restoration into an appropriate shape and volume [1] [2] [3]. 


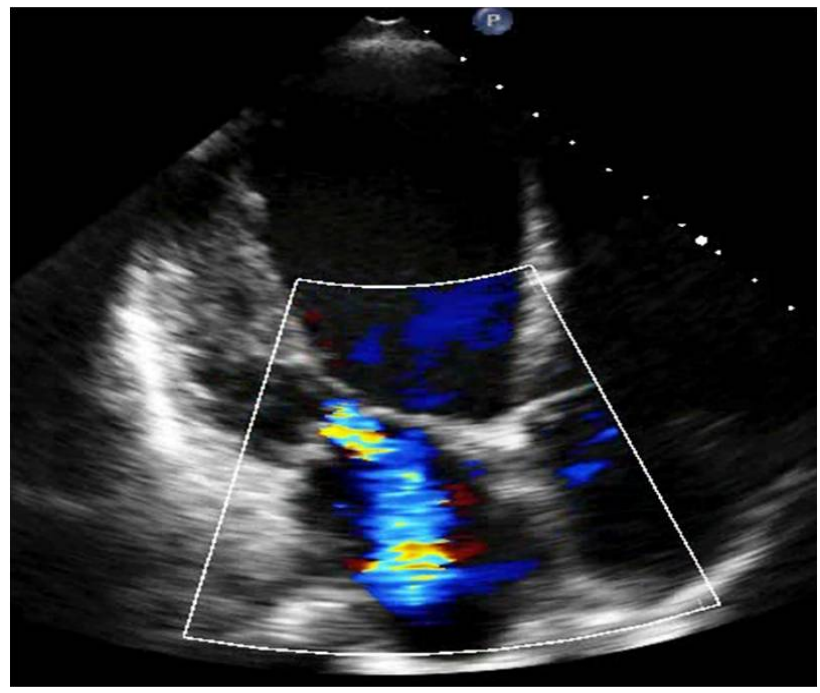

(a)

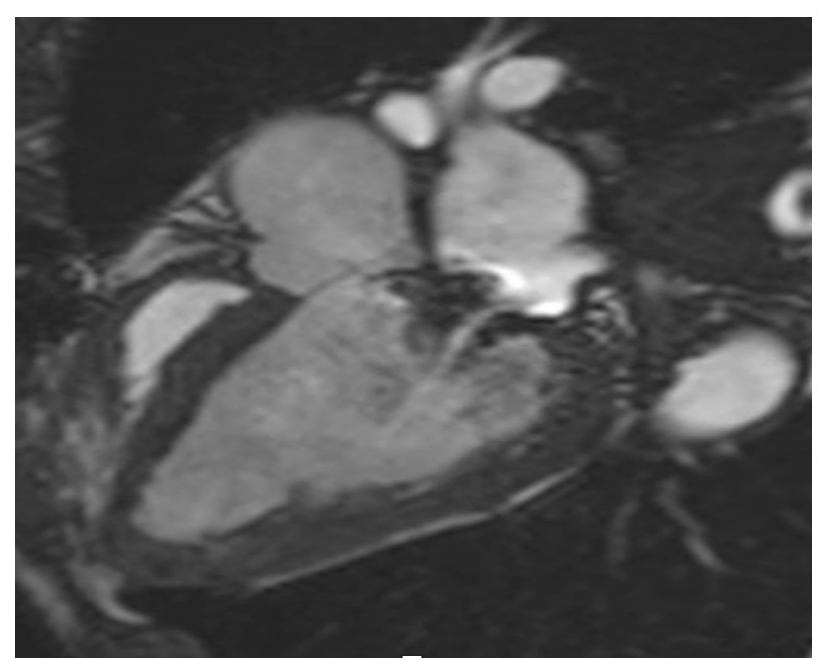

(c)

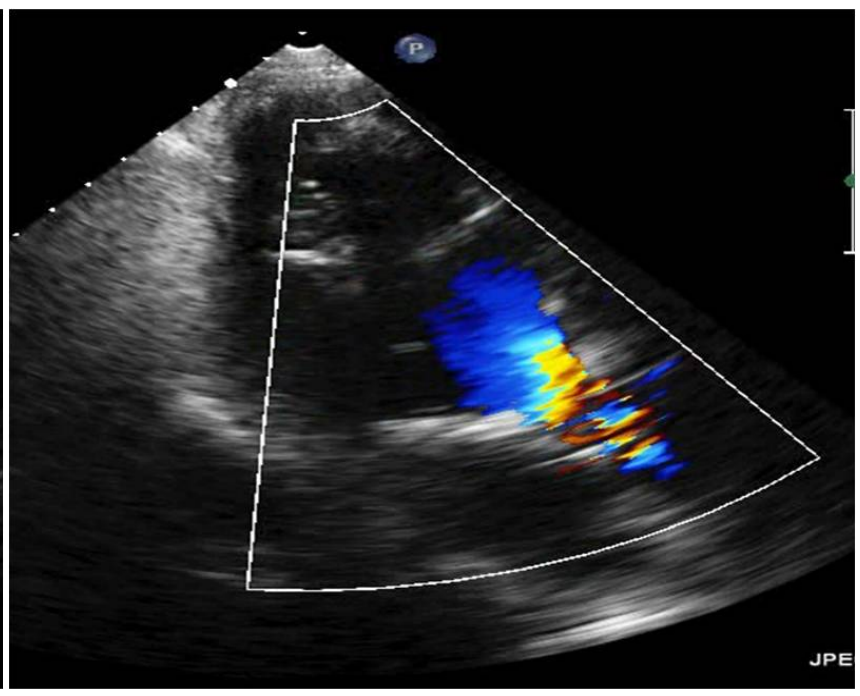

(b)

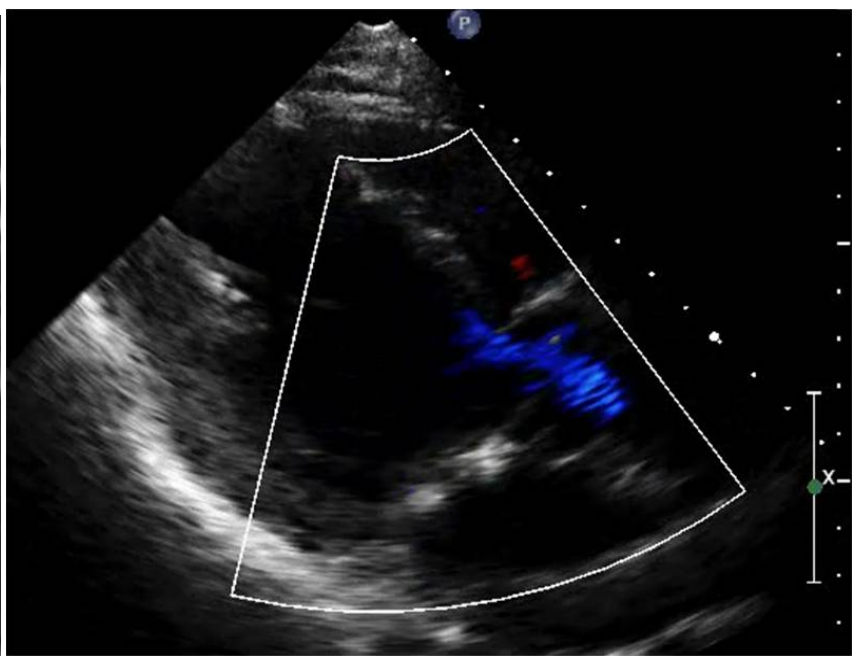

(d)

Figure 3. (a) Preoperative echocardiography showing moderate mitral regurgitation; (b) Echocardiography performed 1 week postoperatively showing no mitral regurgitation; (c) Postoperative magnetic resonance imaging showing improvement of left ventricle (LV) motion and LV shape; (d) Echocardiography performed 1 year postoperatively showing no mitral regurgitation.

Table 1. Data of preoperative and postoperative echocardiography performed 1 week and 1 year after surgery.

\begin{tabular}{cccc}
\hline & Preoperative & $\begin{array}{c}\text { One week } \\
\text { after surgery }\end{array}$ & $\begin{array}{c}\text { One year } \\
\text { after surgery }\end{array}$ \\
\hline LVDd $(\mathrm{mm})$ & 66 & 63 & 63 \\
LVDs (mm) & 61 & 51 & 56 \\
IVST (mm) & 9.4 & 11.6 & 5.6 \\
PWT (mm) & 10.2 & 11.8 & 7.9 \\
LVMI $\left(\mathrm{g} / \mathrm{m}^{2}\right)$ & 221 & 258 & 129 \\
LVEF $(\%)$ & 11 & 31 & 23 \\
LVEDV $(\mathrm{ml})$ & 273 & 160 & 200 \\
LVESV $(\mathrm{ml})$ & 244 & 111 & 154 \\
MR & moderate & none & none \\
\hline
\end{tabular}


ESP could be performed simply because it only requires double-spiral stitches instead of patch materials. A continuous spiral stitch can reconstruct the LV shape both at the short and long axes [4] [5]. Furthermore, the LV was cut to open only 2 to $3 \mathrm{~cm}$ at the scar tissue in the lateral apical wall, resulting in a minimal LV incision. Moreover, papillary muscle approximation can be considered in cases of ischemic MR whose dimension between the papillary muscles is greater than $3 \mathrm{~cm}$, similar to the present case. Restoration of the LV appears to also restore the tethering of the mitral leaflet by surgical reverse remodeling of the LV. MR is treated by papillary muscle approximation to repair the mitral tethering [1] [6]. Moreover, ESP can be carried out without cardiac arrest if LV function is extremely poor to undergo SVR [4] [5].

However, the indication of ESP is limited to selected cases where the infarction area is located in the apex. If the infarction area is broad and located in the other parts of LV, other SVR procedures may be more appropriate [1]. In the present case, because the infarction area was relatively located in the apex, this procedure was preferable. Furthermore, when the ventricular muscle fibers are fragile due to infarction, they are at risk for splitting and cutting [4]. According to Hiraoka et al., patients with extensively thin-walled LV cavities without viable cardiac muscle are not suitable candidates for this technique [4]. Moreover, ESP's long-term postoperative prognosis remains unclear.

Based on the present case, this procedure is expected to have a possibility in thickening of LV wall in an only short term. This strategy might solve recurrent cardiac events due to MR; however, its effectiveness regarding LV wall thickening might be temporary. In order to clarify this matter, further reports are warranted.

\section{Conclusion}

ESP is a less invasive SVR involving a small LV incision and does not require patch materials. Because of papillary muscle approximation, this procedure might be effective in avoiding postoperative cardiac events due to recurrent MR.

\section{Acknowledgements}

We thank the Honyaku center for reviewing and editing the manuscript. We thank our colleagues for helpful comments.

\section{Conflicts of Interest}

The authors declare no conflicts of interest regarding the publication of this paper.

\section{References}

[1] Isomura, T. (2011) Surgical Left Ventricular Reconstruction. General Thoracic and Cardiovascular Surgery, 59, 315-325. https://doi.org/10.1007/s11748-010-0742-4

[2] Menicanti, L., Di Donato, M., Frigiola, A., The RESTORE Group, et al. (2002) 
Ischemic Mitral Regurgitation: Intraventricular Papillary Muscle Imbrication without Mitral Ring during Left Ventricular Restoration. The Journal of Thoracic and Cardiovascular Surgery, 123, 1041-1050. https://doi.org/10.1067/mtc.2002.121677

[3] Buckberg, G.D. and Athanasuleas, C.L. (2009) The STICH Trial: Misguided Conclusions. The Journal of Thoracic and Cardiovascular Surgery, 138, 1060-1064. https://doi.org/10.1016/j.jtcvs.2009.07.015

[4] Hiraoka, A., Kuinose, M., Chikazawa, G., et al. (2011) A Modified Technique of Left Ventricular Restoration: Endoventricular Spiral Plication. Journal of Cardiac Surgery, 26, 261-263. https://doi.org/10.1111/j.1540-8191.2011.01241.x

[5] Hiraoka, A., Kuinose, M., Chikazawa, G., et al. (2012) Endoventricular Spiral Plication for Ischemic Dilated Cardiomyopathy. The Journal of Thoracic and Cardiovascular Surgery, 144, 269-270. https://doi.org/10.1016/j.jtcvs.2012.01.013

[6] Nair, R.U., Williams, S.G., Nwafor, K.U., et al. (2001) Left Ventricular Volume Reduction without Ventriculectomy. The Annals of Thoracic Surgery, 71, 2046-2049. https://doi.org/10.1016/S0003-4975(01)02460-2 